\title{
ON CONTINUABILITY OF SOLUTIONS OF SECOND ORDER DIFFERENTIAL EQUATIONS
}

\author{
T. BURTON AND R. GRIMMER
}

Abstract. An equation (1) $x^{\prime \prime}+a(t) f(x)=0$ together with some generalizations are considered. We give necessary and sufficient conditions in order that (1) have solutions which are not continuable in a certain fashion whenever $a(t)$ becomes negative.

1. Introduction. We consider the equation

$$
x^{\prime \prime}+a(t) f(x)=0 \quad\left({ }^{\prime}=d / d t\right)
$$

where $a$ is continuous for $0 \leqq t<\infty, f$ is continuous for $-\infty<x<\infty$, and $x f(x)>0$ for $x \neq 0$.

In case $a(t)$ is positive, then simple smoothness conditions on $a(t)$ are known which guarantee that all solutions of (1) can be continued for all future time. It is also known (cf. [3] or [5]) that if $a(t)$ is positive and of bounded variation except at one point, then solutions of (1) are not always defined in the future.

The case for $a(t)$ negative at some point is quite different. In [7] (cf. [6]), it is shown that if $a(t)$ is continuous and negative at one point then

$$
x^{\prime \prime}+a(t) x^{2 n+1}=0
$$

(for $n \geqq 1$ ) has solutions which are not defined in the future. Furthermore, this is completely independent of the smoothness of $a(t)$.

The problem of continuability of solutions is of particular importance in the theory of oscillations. During the last several years a number of investigators have attempted to prove that solutions of (1) have arbitrarily large zeros even when $a(t)$ is allowed to become negative for some values of $t$ (cf. [1], [4], [7], [8] and [9]).

In this note we give necessary and sufficient conditions for (1) to have solutions tending monotonically to infinity in finite time whenever $a(t)$ becomes negative at some point. We also show that this result applies to a more general equation (see equation (3)) and that (1) can be used as a comparison equation. Among other things, this shows that attempts to prove that solutions of (1) have arbitrarily

Received by the editors October 20, 1970.

AMS 1970 subject classifications. Primary 34A15, 34C15.

Key words and phrases. Continuability of solutions, second order nonlinear oscillations.

Copyright @ 1971, American Mathematical Society 
large zeros even when $a(t)$ becomes negative should be preceded by continuability results. Although Kiguradze [7] gave some conditions which guaranteed that there exist nontrivial continuable solutions of (2) when $a(t)$ becomes negative, such conditions for (1) are unknown to the authors.

2. Continuability. For convenience in notation, define

$$
F(x)=\int_{0}^{x} f(s) d s .
$$

TheOREM 1. Suppose $a\left(t_{1}\right)<0$ for some $t_{1}>0$. If either

(a) $\int_{0}^{+\infty}[1+F(x)]^{-1 / 2} d x<\infty$ or

(b) $\int_{0}^{-\infty}[1+F(x)]^{-1 / 2} d x>-\infty$,

then (1) has solutions $x(t)$ which are not continuable to $+\infty$.

Proof. Since $a\left(t_{1}\right)<0$ and $a$ is continuous, there are positive numbers $\delta, m$, and $M$ such that $-M \leqq a(t) \leqq-m<0$ if $t_{1} \leqq t \leqq t_{1}+\delta$. In (1), let $x^{\prime}=y$ to obtain the system

$$
x^{\prime}=y, \quad y^{\prime}=-a(t) f(x) .
$$

Assume that condition (a) holds. Denote by $(x(t), y(t))$ a solution of $(1)^{\prime}$ satisfying $x\left(t_{1}\right)=0$ with $y\left(t_{1}\right)$ large and to be determined. So long as $(x(t), y(t))$ is defined on $\left[t_{1}, t_{1}+\delta\right)$ we have both $x(t)$ and $y(t)$ monotonically increasing. From $(1)^{\prime}$ we obtain $y y^{\prime}=-a(t) f(x) x^{\prime}$ which, upon integration, yields $y^{2}(t)-y^{2}\left(t_{1}\right)=-2 \int_{t_{1}}^{t} a(s) f(x(s)) x^{\prime}(s) d s$. Since $x(t)$ is increasing, there is a $\bar{t}$ satisfying $t_{1}<\bar{t}<t$ such that

$$
y^{2}(t)-y^{2}\left(t_{1}\right)=-2 a(\bar{t}) \int_{z\left(t_{1}\right)}^{x(t)} f(u) d u .
$$

As $x\left(t_{1}\right)=0$, we have

$$
y^{2}(t)-y^{2}\left(t_{1}\right)=-2 a(\bar{t}) F(x(t))
$$

so long as $(x(t), y(t))$ is defined and $t_{1}<t<t_{1}+\delta$. Thus,

$$
y(t)=\left[y^{2}\left(t_{1}\right)-2 a(\bar{t}) F(x(t))\right]^{1 / 2}
$$

and so

$$
\left[y^{2}\left(t_{1}\right)+2 m F(x(t))\right]^{1 / 2} \leqq y(t) \leqq\left[y^{2}\left(t_{1}\right)+2 M F(x(t))\right]^{1 / 2} .
$$

Since $x^{\prime}=y$, we have

$$
x^{\prime}(t) \geqq\left[y^{2}\left(t_{1}\right)+2 m F(x(t))\right]^{1 / 2}
$$

or 


$$
\left[y^{2}\left(t_{1}\right)+2 m F(x)\right]^{-1 / 2} d x \geqq d t .
$$

Integrating both sides from $t_{1}$ to $t$ and recalling that $x\left(t_{1}\right)=0$ we have

$$
\int_{0}^{x(t)}\left[y^{2}\left(t_{1}\right)+2 m F(s)\right]^{-1 / 2} d s \geqq t-t_{1} .
$$

Since (a) holds, we may choose $y^{2}\left(t_{1}\right)$ so large that the integral is smaller than $\delta$. It then follows that $x(t) \rightarrow \infty$ before $t$ reaches $t_{1}+\delta$. In order to show that the integral can be made smaller than $\delta$ by taking $y\left(t_{1}\right)$ large, one can proceed as follows. Let $\epsilon>0$ be given. By (a), there exists $X>0$ such that $\int_{X}^{\infty}[1+F(s)]^{-1 / 2} d s<\epsilon$. Write $\mu_{1}$ $=\left[y^{2}\left(t_{1}\right) / 2 m\right]-1$ and agree that $y\left(t_{1}\right)$ will be taken so large that $\mu_{1}>0$. Then

$$
\begin{aligned}
& \int_{0}^{\infty}\left[y^{2}\left(t_{1}\right)+2 m F(s)\right]^{-1 / 2} d s=[2 m]^{-1 / 2} \int_{0}^{\infty}\left[\mu_{1}+1+F(s)\right]^{-1 / 2} d s \\
& =[2 m]^{-1 / 2}\left\{\int_{0}^{x}\left[\mu_{1}+1+F(s)\right]^{-1 / 2} d s+\int_{X}^{\infty}\left[\mu_{1}+1+F(s)\right]^{-1 / 2} d s\right\} \\
& \quad<[2 m]^{-1 / 2}\left\{\int_{0}^{x}\left[\mu_{1}+1+F(s)\right]^{-1 / 2} d s+\epsilon\right\} .
\end{aligned}
$$

Since $X$ is fixed, we may take $y\left(t_{1}\right)$ so large that the integral is smaller than $\epsilon$. This yields the result when $\epsilon$ is chosen so that $2 \epsilon[2 m]^{-1 / 2}<\delta$. The proof is then complete in case (a) holds. If (b) holds, then a similar argument may be given in Quadrant III of the $x y$-plane.

REMARK. It is easy to see how condition (a) relates to equation (2) for $n \geqq 1$ and $a\left(t_{1}\right)<0$. Equation (2) will have many noncontinuable solutions as was shown by Kiguradze [7]. Theorem 1 is a direct generalization of Kiguradze's result. Condition (a) is also related to the hypothesis $\int_{1}^{\infty}[d u / f(u)]<\infty$ which was asked by Bobisud [1] (see also [8]) who allowed $a(t)$ to become negative but restricted his discussion to continuable solutions. In view of our Theorem 1 , it would be interesting to know what conditions should be imposed on (1) to assure the existence of some continuable solutions.

We note the manner in which solutions were shown to tend to infinity in finite time and obtain the following result.

It is known that the only way in which a solution $(x(t), y(t))$ of $(1)^{\prime}$ can fail to be defined past some $T$ is if $\lim _{t \rightarrow T^{-}}\left[x^{2}(t)+y^{2}(t)\right]=+\infty$ (cf. [2, p. 61]). It is easily shown that if $a(t)$ is continuous and nonnegative for all $t \geqq 0$, then there is no $T$ for any solution $(x(t), y(t))$ of $(1)^{\prime}$ satisfying $\lim _{t \rightarrow T^{-}} x^{2}(t)=+\infty$. Thus, the only way in which it is 
possible for solutions to behave as in the proof of Theorem 1 is for $a\left(t_{1}\right)<0$ for some $t_{1}$.

THEOREM 2. Let $a(t)$ be continuous and satisfy $a(t)<0$ on an interval $t_{1} \leqq t<t_{2}$ with $a\left(t_{2}\right) \leqq 0$. Then (1)' has a solution $(x(t), y(t))$ defined for $t=t_{1}$ and satisfying $\lim _{t \rightarrow T^{-}}|x(t)|=+\infty$ for some $T$ satisfying $t_{1}<T$ $\leqq t_{2}$ if and only if either (a) or (b) holds.

Proof. By Theorem 1 and its proof we know that such solutions exist if $a\left(t_{1}\right)<0$ and if (a) or (b) holds.

Assume that such solutions exist. To be definite, suppose that there is a solution $(x(t), y(t))$ of (1) defined at $t=t_{1}$ and satisfying $\lim _{t \rightarrow T^{-}} x(t)=+\infty$ for $t_{1}<T \leqq t_{2}$. Thus, $(x(t), y(t))$ is defined on the interval $\left[t_{1}, T\right)$. We shall show that (a) holds.

Since $x^{\prime}=y$ and since $x(t) \rightarrow \infty$, there is some $\bar{t}$ satisfying $t_{1} \leqq \bar{t}<T$ for which $x(t)>0$ and $y(t)>0$ for $\bar{t} \leqq t<T$. As in the proof of Theorem 1 , there are constants $m$ and $M$ such that $-M \leqq a(t) \leqq-m$ for $\bar{t} \leqq t \leqq T$ where $m \geqq 0$ since $a\left(t_{2}\right) \leqq 0$ and

$$
\begin{aligned}
& {\left[y^{2}(\bar{t})+2 m\{F(x(t))-F(x(\bar{t}))\}\right]^{1 / 2} \leqq y(t)} \\
& \left.\leqq\left[y^{2}(\bar{t})+2 M\{F(x(t))-F(x(\bar{t}))\}\right]\right]^{1 / 2}
\end{aligned}
$$

for $\bar{t} \leqq t<T$. Again, $x^{\prime}=y$ so

$$
x^{\prime}(t) \leqq\left[y^{2}(\bar{t})+2 M\{F(x(t))-F(x(\bar{t}))\}\right]^{1 / 2}
$$

or

$$
\left[y^{2}(\bar{t})+2 M\{F(x(t))-F(x(\bar{t}))\}\right]^{-1 / 2} d x \leqq d t .
$$

An integration from $\bar{t}$ to $t$ yields

$$
\int_{x(\bar{t})}^{x(t)}\left[y^{2}(\bar{t})+2 M\{F(s)-F(x(\bar{t}))\}\right]^{-1 / 2} d s \leqq t-\bar{t} .
$$

Now $F(x)$ is an increasing function for $x>0$ and we know that $x(t)$ is increasing. Thus, since $y^{2}(\bar{t})>0$, the integrand is defined. Since $x(t) \rightarrow \infty$ as $t \rightarrow T^{-}$, we see that

$$
\int_{x(\bar{i})}^{\infty}\left[y^{2}(\bar{t})+2 M\{F(s)-F(x(\bar{t}))\}\right]^{-1 / 2} d s<\infty .
$$

If we write $w(\bar{t})=\left(y^{2}(\bar{t}) / 2 M\right)-F(x(\bar{t}))$, then

$$
\int_{x(\bar{b})}^{\infty}[w(\bar{t})+F(s)]^{-1 / 2} d s<\infty .
$$


If $w(\bar{t}) \leqq 1$, then $[w(\bar{t})+F(s)]^{-1 / 2} \geqq[1+F(s)]^{-1 / 2}$ and so (a) holds. Now suppose that $w(\bar{t})>1$. Then

$$
\begin{aligned}
{[w(\bar{t})]^{-1 / 2} } & \int_{x(\bar{l})}^{\infty}[1+\{1 / w(\bar{t})\} F(s)]^{-1 / 2} d s \\
& >[w(\bar{t})]^{-1 / 2} \int_{x(\bar{t})}^{\infty}[1+F(s)]^{-1 / 2} d s .
\end{aligned}
$$

Since the first integral converges, so does the second and hence (a) holds.

If $x(t) \rightarrow-\infty$ as $t \rightarrow T^{-}$, then a similar proof may be carried out in Quadrant III of the $x y$-plane showing that (b) holds, thus completing the proof.

We now show that our results hold for the apparently more general equation

$$
x^{\prime \prime}+q(t) x^{\prime}+a(t) f(x)=0
$$

in which $a$ and $f$ are as before and $q$ is continuous for $0 \leqq t<\infty$.

If we let

$$
r(t)=\exp \int_{0}^{t} q(s) d s
$$

then (3) may be written as

$$
\left(r(t) x^{\prime}\right)^{\prime}+r(t) a(t) f(x)=0 .
$$

Now let

$$
s=\int_{0}^{t}[d u / r(u)] \text { and } x(t)=y(s)
$$

so that (4) becomes

$$
\ddot{y}(s)+r^{2}(t) a(t) f(y(s))=0
$$

where $\cdot=d / d s$.

Now a $t$ interval $\left[t_{1}, t_{2}\right]$ on which $a(t)<0$ is transformed to an $s$ interval $\left[s_{1}, s_{2}\right]$ where $s_{i}=\int_{0}^{t_{i}}[d u / r(u)]$. Thus, if $a(t)$ is negative on $\left[t_{1}, t_{2}\right]$, then $r^{2}(t(s)) a(t(s))$ is negative on $\left[s_{1}, s_{2}\right]$. Our arguments may now be applied to equation (5). for

Finally, we observe that (1) can be used as a comparison equation

$$
x^{\prime \prime}+a(t) g\left(x, x^{\prime}, t\right)=0
$$


in which $g$ is continuous for $-\infty<x<\infty,-\infty<x^{\prime}<\infty$, and $0 \leqq t<\infty$, while $a(t)$ is continuous on $0 \leqq t<\infty$.

TheOREM 3. Let $a\left(t_{1}\right)<0$ for some $t_{1}>0$. Suppose there exists an increasing function $f$ which is continuous for $-\infty<x<\infty$ and $x f(x)>0$ if $x \neq 0$. In addition, suppose there exists $\delta_{1}>0$ and $N>0$ such that for $t_{1} \leqq t \leqq t_{1}+\delta_{1}$ either

(A) $f(x) \leqq g(x, y, t)$ for $x>N$ and $y>N$, or

(B) $g(x, y, t) \leqq f(x)$ for $x<-N$ and $y<-N$.

If (A) and (a) hold or if (B) and (b) hold, then (6) has solutions $x(t)$ satisfying $|x(t)| \rightarrow \infty$ as $t \rightarrow T_{1}^{-}$for some $T_{1}>t_{1}$.

Proof. Suppose that (A) and (a) hold. From the proof of Theorem 1 , there exists a solution $(x(t), y(t))$ of $(1)^{\prime}$ satisfying $x\left(t_{1}\right)=0, y\left(t_{1}\right)$ $>N$, and $x(t) \rightarrow+\infty$ as $t \rightarrow T-$ for $t_{1}<T<t_{1}+\delta$ where $\delta$ is from the proof of Theorem 1 and $\delta$ may be chosen to satisfy $\delta<\delta_{1}$. Now on the interval $\left[t_{1}, T\right), y(t)$ is also increasing. Thus, there is a number $t_{3}$ satisfying $t_{1}<t_{3}<T$ such that $x\left(t_{3}\right)>N$. In the $t x$-plane, consider a solution of (6), say $\bar{x}(t)$ satisfying $\bar{x}\left(t_{3}\right)>x\left(t_{3}\right)$ and $\bar{x}^{\prime}\left(t_{3}\right)>y\left(t_{3}\right)$. So long as $\bar{x}(t)$ is defined on $\left[t_{3}, T\right)$, we have $\bar{x}(t)>N$ and $\bar{x}^{\prime}(t)>N$. We now show that $\bar{x}(t)>x(t)$ for all $t \geqq t_{3}$ for which $\bar{x}(t)$ is defined. If this is false, then there exists $t_{4}>t_{3}$ with $\bar{x}\left(t_{4}\right)=x\left(t_{4}\right)$ and $\bar{x}(t)>x(t)$ on $\left[t_{3}, t_{4}\right)$. Now $\bar{x}(t)>N$ and $\bar{x}^{\prime}(t)>N$ on $\left[t_{3}, t_{4}\right)$ and so

$$
\begin{aligned}
\bar{x}^{\prime \prime}(t)-x^{\prime \prime}(t) & =-a(t) g\left(\bar{x}(t), \bar{x}^{\prime}(t), t\right)+a(t) f(x(t)) \\
& \geqq-a(t) f(\bar{x}(t))+a(t) f(x(t))>0
\end{aligned}
$$

since $f$ is increasing, $a(t)<0$, and $\bar{x}(t)>x(t)$. Thus, $\bar{x}^{\prime}(t)-x^{\prime}(t)$ is increasing on $\left[t_{3}, t_{4}\right)$ and hence, as $\bar{x}^{\prime}\left(t_{3}\right)-x^{\prime}\left(t_{3}\right)>0$ we have $\bar{x}^{\prime}(t)-x^{\prime}(t)$ $>0$ on $\left[t_{3}, t_{4}\right)$. But then $\bar{x}(t)-x(t)$ is increasing on $\left[t_{3}, t_{4}\right)$ so $\bar{x}\left(t_{4}\right)-x\left(t_{4}\right)$ $>\bar{x}\left(t_{3}\right)-x\left(t_{3}\right)>0$. Since $x(t) \rightarrow \infty$ as $t \rightarrow T^{-}$, there is some $T_{1}$ satisfying $t_{3}<T_{1} \leqq T$ with $\bar{x}(t) \rightarrow \infty$ as $t \rightarrow T_{1}^{-}$. Thus, the proof is complete for the case in which (A) and (a) hold. The proof in case (B) and (b) hold is similar.

We leave the formulation of the comparison result corresponding to Theorem 2 to the reader.

\section{REFERENCES}

1. L. E. Bobisud, Oscillations of nonlinear second-order equations, Proc. Amer. Math. Soc. 23 (1969), 501-505. MR 40 \#448.

2. E. A. Coddington and N. Levinson, Theory of ordinary differential equations, McGraw-Hill, New York, 1955. MR 16, 1022.

3. C. V. Coffman and D. F. Ulrich, On the continuation of solutions of a certain nonlinear differential equation, Monatsh. Math. 71 (1967), 385-392. MR 37 \#3078. 
4. L. Erbe, Oscillation theorems for second order nonlinear differential equations, Proc. Amer. Math. Soc. 24 (1970), 811-814. MR 40 \#5973.

5. S. P. Hastings, Boundary value problems in one differential equation with a discontinuity, J. Differential Equations 1 (1965), 346-369. MR 31 \#954.

6. J. W. Heidel, Uniqueness, continuation, and nonoscillation for a second order nonlinear differential equation, Pacific J. Math. 32 (1970), 715-721.

7. I. T. Kiguradze, $A$ note on the oscillation of solutions of the equation $u^{\prime \prime}+$ $a(t)|u|{ }^{n} \operatorname{sgn} u=0$, Časopis Pěst. Mat. 92 (1967), 343-350. (Russian) MR $36 \# 4064$.

8. G. G. Legatos and A. G. Kartsatos, Further results on oscillation of solutions of second order equations, Math. Japon. 14 (1969), 67-73.

9. P. Waltman, An oscillation criterion for a nonlinear second order equation, J. Math. Anal. Appl. 10 (1965), 439-441. MR 30 \#3265.

Southern Illinois University, Carbondale, Illinois 62901 